\title{
Extra dimensions hypothesis in high energy physics
}

\author{
Igor Volobuev ${ }^{1, \star}$, Eduard Boos ${ }^{1, \star \star}$, Viacheslav Bunichev ${ }^{1, \star \star \star}$, Maxim Perfilov ${ }^{1, \star \star \star \star}$, and \\ Mikhail Smolyakov ${ }^{1, \dagger}$
}

${ }^{1}$ D.V. Skobeltsyn Institute of Nuclear Physics, Lomonosov Moscow State University, 119991 Moscow, Russia

\begin{abstract}
We discuss the history of the extra dimensions hypothesis and the physics and phenomenology of models with large extra dimensions with an emphasis on the RandallSundrum (RS) model with two branes. We argue that the Standard Model extension based on the RS model with two branes is phenomenologically acceptable only if the inter-brane distance is stabilized. Within such an extension of the Standard Model, we study the influence of the infinite Kaluza-Klein (KK) towers of the bulk fields on collider processes. In particular, we discuss the modification of the scalar sector of the theory, the Higgs-radion mixing due to the coupling of the Higgs boson to the radion and its KK tower, and the experimental restrictions on the mass of the radion-dominated states.
\end{abstract}

\section{Introduction}

The hypothesis about the existence of extra space-time dimensions is discussed in theoretical physics for more than a century. There is a good reason to believe that this hypothesis grew from the ideas formulated by Bernhard Riemann in his famous lecture "On the Hypotheses which lie at the Bases of Geometry" [1]. In this lecture Riemann formulated the notion of n-dimensional space and suggested that the space-time geometry at microscopic distances may be very different from the macroscopic one.

The first attempt to construct a physical theory in a space-time with extra dimensions was made by Gunnar Nordström in 1914 in his work "On the possibility of unifying the electromagnetic and the gravitational fields" [2], where he tried to unify a relativistic scalar gravity theory, which he was developing at that time, with electrodynamics. In fact, the construction of Nordström was very similar to the present-day braneworld models: our four-dimensional world was embedded in a five-dimensional one as a submanifold. Though Nordström's scalar theory of gravity was a very important step in constructing Einstein's general theory of relativity, it was forgotten soon after the latter proved to be a success. Correspondingly, the idea of extra dimensions was abandoned for five years to be rediscovered by Theodor Kaluza and implemented in the framework of Einstein's general theory of relativity.

\footnotetext{
${ }^{\star}$ e-mail: volobuev@ theory.sinp.msu.ru

$\star \star$ e-mail: boos@theory.sinp.msu.ru

$\star \star \star$ e-mail: bunichev@ theory.sinp.msu.ru

$\star \star \star \star$ e-mail: perfilov@theory.sinp.msu.ru

†e-mail: smolyakov@theory.sinp.msu.ru
} 


\section{Kaluza-Klein theory}

The original theory of Kaluza [3] was a pure gravity in space-time $E=M^{4} \times R^{1}$, where $M^{4}$ is four-dimensional space-time. If we denote the coordinates in $E$ by $X^{N}=\left(x^{v}, y\right), \quad N=0,1, \cdots 4$, $v=0,1, \cdots 3$, the standard gravitational action in this space can be written as

$$
S=\frac{1}{16 \pi \hat{G}} \int_{E} \hat{R} \sqrt{-g} d^{5} X
$$

where $\hat{G}$ is the five-dimensional gravitational constant and $\hat{R}$ is the scalar curvature of the metric $g_{M N}, \operatorname{sign}(g)=(-,+\cdots+)$. Kaluza suggested that this five-dimensional theory reproduced fourdimensional gravity theory coupled to electromagnetic field, if the fields of the original theory did not depend on the coordinate of the extra dimension.

The next step in theories with extra dimensions was made by Oskar Klein [4], who proposed to consider the vacuum configuration of the five-dimensional metric and to include scalar, spinor and vector fields in the multidimensional theory from the very beginning. He showed that, in this case, for every four-dimensional field there exists a tower of fields with the same quantum numbers and the masses of the order of the inverse size of the extra dimension, which are called nowadays the Kaluza-Klein modes.

Finally, in paper [5] by Einstein and Bergmann it was shown that the unobservability of the extra dimension could be explained by its compactness, i.e. that it is the circle $S^{1}$ instead of $R^{1}$, and extremely small size, - of the order of the Planck length $L_{P l}=1 / M_{P l}$.

Let us consider the Kaluza-Klein hypothesis in more detail. To isolate the physical degrees of freedom in this theory, it is useful to consider the linearized theory

$$
g_{M N}(x, y)=\eta_{M N}+\kappa h_{M N}(x, y), \quad \kappa=\sqrt{16 \pi \hat{G}}
$$

This theory is a gauge theory of tensor field with gauge transformations generalizing those of classical electrodynamics, which allows one to impose a convenient gauge condition and to obtain the linearized equations of motion. In the case, where the energy-momentum tensor of matter is equal to zero, these equations take the form

$$
\partial^{P} \partial_{P} h_{M N}(x, y)=\left(\square+\frac{\partial^{2}}{\partial y^{2}}\right) h_{M N}(x, y)=0 .
$$

The corresponding eigenvalue problem for the mass spectrum of the gravitational field fluctuations looks as follows

$$
\frac{\partial^{2}}{\partial y^{2}} h_{M N}(x, y)=-m^{2} h_{M N}(x, y)
$$

Thus, the mass spectrum can be found to be

$$
m_{n}=\frac{2 \pi n}{L}
$$

L denoting the size of the extra dimension, i.e. the circumference of $S^{1}$. It includes the massless zero modes, for which $\mathrm{n}=0$ and whose wave functions do not depend on the coordinate of the extra dimension, and the higher Kaluza-Klein modes that are very heavy, because the size of the extra dimension should be of the order of the Planck length to provide for the unobservability of the extra dimension. 
The zero mode five-dimensional metric $g_{M N}$ can be decomposed into the four-dimensional part and the extra components as

$$
g_{M N}=\left(\begin{array}{cc}
\gamma_{\mu v}+\phi A_{\mu} A_{v} & \phi A_{\mu} \\
\phi A_{v} & \phi
\end{array}\right), \quad \phi=g_{44}, \phi A_{\mu}=g_{\mu 4} .
$$

Since $g_{M N}$ does not depend on $y$, which Kaluza called the "cylindricity condition", the scalar curvature $\hat{R}$ can be expressed as

$$
\begin{aligned}
\hat{R} & =R_{(4)}-\frac{1}{4} F_{\mu \nu} F^{\mu v} \phi^{-1}-\frac{1}{2} \phi^{-2} \partial_{\mu} \phi \partial^{\mu} \phi \\
F_{\mu \nu} & =\partial_{\mu} A_{\nu}-\partial_{\nu} A_{\mu},
\end{aligned}
$$

$R_{(4)}$ being the scalar curvature of the four-dimensional space-time $M^{4}$ with metric $\gamma_{\mu \nu}$.

In the original paper by Kaluza the field $A_{\mu}$ was interpreted as electromagnetic field. But the subsequent studies showed that this interpretation failed.

The size $L$ of the extra dimension being of the order of the Planck length, the Kaluza-Klein hypothesis leads to the conclusion that the observed fields (i.e. the fields of the Standard Model) should be the so called zero modes, i.e. should not depend on the coordinates of extra dimensions. Moreover, for each observed field there should exist a tower of fields with masses of the order of the Planck mass $M_{P l} \sim 1 / L_{P l}$, which cannot be observed at the energies, which are available for experiments nowadays.

Nevertheless, even in this case there remain relations between the multidimensional and the fourdimensional theories, which can manifest themselves as certain symmetries of the reduced fourdimensional theories and the values of their coupling constants. In particular, an important point is the relation between the Planck mass in four dimensions $M_{P l}$ and the fundamental energy scale $M$ of multidimensional space-time

$$
M_{P l}^{2}=M^{3} L
$$

which can be obtained for the zero modes of gravitational field from action (1) in space-time $E=$ $M^{4} \times S^{1}$ just by integrating it over the circle $S^{1}$.

\section{Large extra dimensions}

The interpretation of a theory in a space-time with extra dimensions in terms of a four-dimensional one was given the name of dimensional reduction. The presence of boson fields in the multidimensional theory led to the idea to explain the factorized structure of the multidimensional space-time dynamically as a result of interaction of gravity with gauge and scalar fields. This procedure was called spontaneous compactification. The attempts to construct the Standard Model along these lines showed that large extra dimensions were needed [6], which was obviously in contradiction with their unobservability.

A solution to this problem was found in 1983 by Rubakov and Shaposhnikov, who put forward a new scenario for Kaluza-Klein theories based on the idea of localization of states [7]. They considered a real Higgs field in the flat 5-dimensional space-time and found the exact solution connecting the two disjoint vacua. The energy density of the solution is localized in the vicinity of the 3-dimensional hypersurface $x^{4}=0$ and has a width of the order of the inverse mass of the Higgs field. Such an object is called a thick domain wall and serves as a potential well for the fluctuations of the Higgs field.

It has also been shown that there are only two modes of the fluctuations that are localized on the domain wall. Moreover, the interaction of these modes cannot produce unlocalized modes, if the 
center of mass energy is less than a certain energy scale proportional to the Higgs mass. Thus, for sufficiently small energies the theory describes a 4-dimensional world, although the whole world is, in fact, 5-dimensional.

This model suggests a possibility for extra dimensions to be unobservable, if the fields of the Standard Model are localized by a certain mechanism on a domain wall in multidimensional spacetime. The corresponding localization mechanism turned out to be rather simple for spinors, but a plausible mechanism for gauge fields has not been found yet.

If the thickness of the domain wall goes to zero, it turns into a three-dimensional surface in the multidimensional space, on which the field of the Standard Model are presumably trapped; such an object is called a membrane, or just a "brane". There are indications that this scenario can be implemented in the theory of superstrings.

It is clear that within the framework of this scenario extra dimensions may be much larger, than the Planck length (or even infinite). Based on this assumption, in paper [8] a single brane without tension (i.e. energy density) in a space-time with an arbitrary number of compact extra dimensions was considered. In this case relation (6) between the fundamental multidimensional energy scale and the four-dimensional one, which for $d$ extra dimensions looks like

$$
M_{P l}^{2}=M^{d+2} L^{d}
$$

yields that the fundamental energy scale in multidimensional space (in the bulk) $M$ may be much less than the Planck mass in four dimensions $M_{P l}$, if the volume of the space of extra dimensions $L^{d}$ is large. In other words, gravity in multidimensional space-time becomes "strong" not at the energies of the order of $10^{19} \mathrm{GeV}$, but at much lower energies, maybe of the order of $1-10 \mathrm{TeV}$. Thus, the scenario provides a solution to the hierarchy problem: it gives a strong gravity in the multidimensional space-time and a weak gravity on the brane. The effects due to the interaction of this "strong" gravity with the fields of the Standard Model could be observable already at the energies, which are available at the existing colliders.

A flaw of this approach is the approximation of the zero brane tension, which is rather too rough: it turns out that the proper gravitational field of the brane cannot be taken into account perturbatively at all. In paper [9] an exact solution for two branes interacting with gravity in five-dimensional spacetime was found, which allows one to estimate the influence of the proper gravitational field of the brane on these results. First of all, it turned out that, if extra dimensions are compact, there should exist at least two branes. A similar solution for two branes in space-time with two extra dimenions with a similar form of metric was later found in [10]. In what follows we will discuss in more detail the five-dimensional model, which turned out to be more interesting.

\section{The Randall-Sundrum model}

The model is called the Randall-Sundrum model and is similar to the original Kaluza-Klein theory in the sense that it is a gravity theory in 5-dimensional space-time $E=M^{4} \times S^{1}$ with coordinates $X^{M}=\left(x^{\mu}, y\right)$, the circle $S^{1}$ now being of circumference $2 L$. But unlike the original Kaluza-Klein theory the gravity interacts with two 3-branes. Thus, the action of the system is

$$
S=\int d^{4} x \int_{-L}^{L} d y\left(2 M^{3} \hat{R}-\Lambda\right) \sqrt{-g}-\lambda_{1} \int_{y=0} \sqrt{-\tilde{g}} d^{4} x-\lambda_{2} \int_{y=L} \sqrt{-\tilde{g}} d^{4} x,
$$

where $M$ is the fundamental 5-dimensional energy scale, $\Lambda$ is the cosmological constant in 5dimensional space-time, $\tilde{g}_{\mu \nu}$ is the metric induced on the branes, $\tilde{g}=\operatorname{det} \tilde{g}_{\mu \nu}$, and the subscripts 1,2 distinguish the branes. Moreover, the 5-metric $g$ must be invariant under the reflection $\left(x^{\mu}, y\right) \rightarrow\left(x^{\mu},-y\right)$, 
which is equivalent to identifying the points $\left(x^{\mu}, y\right)$ and $\left(x^{\mu},-y\right)$ of $S^{1}$ and reducing it to the orbifold $S^{1} / Z_{2}$.

The ansatz for the metric, which preserves the Poincaré invariance on every hypersurface $y=$ const, looks like

$$
d s^{2}=e^{-2 \sigma(y)} \eta_{\mu \nu} d x^{\mu} d x^{v}+d y^{2} \equiv \gamma_{M N}(y) d x^{M} d x^{N},
$$

When it is substituted into Einstein equations following from action (7), they reduce to

$$
\begin{aligned}
\left(\frac{d \sigma}{d y}\right)^{2} & =-\frac{\Lambda}{24 M^{3}} \\
6 \frac{d^{2} \sigma}{d y^{2}} & =\frac{1}{2 M^{3}}\left(\lambda_{1} \delta(y)+\lambda_{2} \delta(y-L)\right) .
\end{aligned}
$$

The solution to these equation with the orbifold symmetry is

$$
\begin{aligned}
\sigma(y) & =k|y|+c, \quad \Lambda=-24 M^{3} k^{2} \\
\lambda_{1} & =-\lambda_{2}=24 M^{3} k,
\end{aligned}
$$

where $k$ is a new parameter of the dimension of mass, which can be interpreted as the inverse effective thickness of the branes, and $\mathrm{c}$ is an integrations constant. The choice of this constant is equivalent to a choice of the coordinates $\left\{x^{\mu}\right\}$ on the branes, and we will keep it arbitrary for the moment.

It is worth noting that the space $E$ with metric (8) and $\sigma$ given by solution (9) is a piece of fivedimensional anti-de Sitter space in horospherical coordinates [11] and that brane 1 has a positive energy density, whereas brane 2 has a negative one.

Metric (8) with $\sigma$ given by equation (9) is taken to be the vacuum of the theory, and the physical fields are fluctuations $h_{M N}(x, y)$ above this vacuum, which are treated perturbatively:

$$
g_{M N}(x, y)=\gamma_{M N}(y)+\frac{1}{\sqrt{2 M^{3}}} h_{M N}(x, y) .
$$

If we substitute this representation into action (7) and keep the terms of zero order in $M$, we get a free action for the fluctuations of the gravitational field. It is a common knowledge that linearized gravity is a gauge theory, and the corresponding gauge transformations can be used to isolate the physical degrees of freedom of the model. One can prove that there exist a gauge, where

$$
h_{\mu 4}=0, h_{44}=h_{44}(x) \equiv \phi(x) .
$$

The scalar field $\phi(x)$ is called the radion field. With the help of a substitution including this field one can extract a transverse-traceless tensor field $b_{\mu \nu}(x, y)$ from the tensor field $h_{\mu \nu}(x, y)$. The transversetraceless tensor field $b_{\mu \nu}(x, y)$ and the scalar radion field $\phi(x)$ are the physical degrees of freedom of the Randall-Sundrum model and diagonalize its second variation Lagrangian. One can also prove that the radion field is massless.

To understand the physical meaning of the radion field let us calculate the distance between the branes along a geodesic $x=$ const:

$$
l=\int_{0}^{L} \sqrt{d s^{2}} \sim \int_{0}^{L}\left(1+\frac{1}{2 \sqrt{2 M^{3}}} h_{44}\right) d y=L\left(1+\frac{1}{2 \sqrt{2 M^{3}}} \phi(x)\right) .
$$

This formula means that the field $\phi(x)$ describes local variations of the inter-brane distance, i.e. oscillations of the branes with respect to each other. 
The transverse-traceless tensor field $b_{\mu \nu}(x, y)$ can be expanded as

$$
b_{\mu \nu}(x, y)=\sum_{n} b_{\mu \nu}^{(n)}(x) \psi_{n}(y)
$$

where $b_{\mu \nu}^{(n)}(x)$ are four-dimensional fields with spin 2 and certain masses $m_{n}$. The latter and the wave functions of the modes $\psi_{n}(y)$ are defined by the equation

$$
\left[\frac{1}{2} \frac{d^{2}}{d y^{2}}+2 k(\delta(y)-\delta(y-L))-2 k^{2}\right] \psi_{n}(y)=\frac{m_{n}^{2}}{2} e^{2 \sigma} \psi_{n}(y) .
$$

This equation can be solved exactly, the wave function of the zero mode being proportional to $\exp (-2 \sigma)$ and those of massive modes being expressible in terms of Bessel and Neumann functions.

Now we are in a position to find the relation between the fundamental 5-dimensional energy scale $M$ and the Planck mass. To this end we have to calculate the curvature of the metric

$$
d s^{2}=e^{-2 \sigma(y)} \bar{g}_{\mu \nu} d x^{\mu} d x^{\nu}+d y^{2}
$$

with $\bar{g}_{\mu \nu}=\eta_{\mu \nu}+h_{\mu \nu}(x)$ and integrate it over the extra dimension. Since the wave function of the massless graviton is proportional to $\exp (-2 \sigma)$, this metric corresponds to four-dimensional gravity only and integrating it over the extra dimension gives an effective action for 4-dimensional gravity

$$
S_{e f f}=2 M^{3} \int_{-L}^{L} d y e^{-2(k|y|+c)} \int R(\bar{g}) \sqrt{-\bar{g}} d^{4} x=2 M^{3}\left(1-e^{-2 k L}\right) e^{-2 c} \int d^{4} x R(\bar{g}) \sqrt{-\bar{g}},
$$

which has to coincide with the Hilbert-Einstein action for the metric $\bar{g}_{\mu v}$. But since we have two branes, we have to realize, to which brane this metric corresponds.

If we are on the brane at $y=0$, the metric on the brane induced from metric (15) coincides with $\bar{g}_{\mu \nu}$ only if $c=0$. This leads to the following relation between the energy scales

$$
M_{P l}^{2}=M^{3} \frac{1-e^{-2 k L}}{k} .
$$

Thus, if we live on the brane at $y=0$, relation (17) demands that the fundamental five-dimensional energy scale $M$ and the parameter $k$ should be of the order of $M_{P l}, \quad M \sim k \sim M_{P l}$. This means that for the observer on brane 1 the five-dimensional gravity is as weak, as the four-dimensional one.

If we are on the brane at $y=L$, the metric on the brane induced from metric (15) coincides with $\bar{g}_{\mu v}$ only if $c=-k L$. In this case formula (16) gives us the following relation between the Planck mass $M_{P l}$ and the fundamental five-dimensional energy scale $M$ :

$$
M_{P l}^{2}=M^{3} \frac{e^{2 k L}-1}{k} .
$$

Obviously, both relations (17) - (18) reduce to (6) (with $L$ replaced by $2 L$ ) for $k \rightarrow 0$, i.e. when the effective thickness of the brane goes to infinity and the very notion of a brane is meaningless.

If our world is situated on the brane at $y=L$, we can put $k L \sim 35$ in formula (18), which gives that the funamental five-dimensional energy scale $M$ and the parameter $k$ may be of the order of $1 \mathrm{TeV}$. That is the hierarchy problem of the gravitational interaction is solved for the observer on brane 2: the energy scale of the five-dimensional gravity comes down to the electroweak energy scale, and the gravity on the brane becomes weak due to the exponential warp factor in relation (18). Thus, fivedimensional gravity in the Randall-Sundrum model looks different for observers on different branes. 
In what follows we will assume that we live on the brane at $y=L$. If we consider the interaction of the physical fields with matter on this brane, we will find that the coupling of the massive tensor fields to the energy-momentum tensor of SM is of the order of inverse $\mathrm{TeV}$. The coupling of the radion field $\phi(x)$ to the trace of the energy-momentum tensor is also of the same order, i.e. much stronger than that of the zero mode of the tensor field. Thus, a scalar gravity is realized on brane 2 , which is phenomenologically unacceptable. A way to cure this problem without violating the solution of the hierarchy problem is to give a mass to the radion. It turns out that this is equivalent to stabilizing the distance between the branes.

\section{Stabilized Randall-Sundrum model}

Mechanisms for stabilizing the Randall-Sundrum model were put forward in papers $[12,13]$. Both of them employ a five-dimensional scalar field to stabilize the inter-brane distance, but the mechanism of paper [13] seems to be preferable because it is based on an exact solution for gravity interacting with two branes and a scalar field in five-dimensional space-time. The physical degrees of freedom of models stabilized by this mechanism were isolated in paper [14]. They are the transverse-traceless tensor fields $b_{\mu}^{n}(x), n=0,1, \cdots$ with masses $m_{n}\left(m_{0}=0\right)$ and scalar fields $\varphi_{n}(x), n=1,2, \cdots$ with masses $\mu_{n}$. The coupling constants to matter of the massive tensor fields remain essentially the same as in the unstabilized model, i.e. of the order of $1 \mathrm{TeV}^{-1}$. The couplings of the scalar fields are also defined by their wave functions and are essentially of the same order.

If we consider the extension of the Standard Model based on the stabilized Randall-Sundrum model, where the SM fields are localized on the brane at $y=L$, we see that the vacua of the stabilized Randall-Sundrum model and the SM are independent. If we assume that this brane world model is an indivisible theory, it is reasonable to believe that there should be a common interconnected vacuum solution for all these fields. To this end we modify action (7) so that it contains a quartic interaction of the stabilizing scalar field and of the Higgs field, which would connect the stabilization of the extra dimension size and the SM spontaneous symmetry breaking.

The modified action looks like

$$
S=S_{g}+S_{\phi+S M}
$$

where $S_{g}$ and $S_{\phi+S M}$ are given by

$$
\begin{aligned}
S_{g} & =2 M^{3} \int d^{4} x \int_{-L}^{L} d y R \sqrt{-g} \\
S_{\phi+S M} & =\int d^{4} x \int_{-L}^{L} d y\left(-\frac{1}{2} g^{M N} \partial_{M} \phi \partial_{N} \phi-V(\phi)\right) \sqrt{-g}- \\
& -\int_{y=0} \sqrt{-\tilde{g}} V_{1}(\phi) d^{4} x+\int_{y=L} \sqrt{-\tilde{g}}\left(-V_{2}(\phi)+L_{S M-H P}+L_{i n t}(\phi, H)\right) d^{4} x .
\end{aligned}
$$

Here $M$ is the fundamental five-dimensional energy scale, $V(\phi)$ is a bulk stubilizing scalar field potential and $V_{1,2}(\phi)$ are brane scalar field potentials, $\tilde{g}=\operatorname{det} \tilde{g}_{\mu \nu}$, and $\tilde{g}_{\mu \nu}$ denotes the metric induced on the branes. The SM fields are localized on the brane at $y=L$, and the Lagrangian $L_{S M-H P}$ is the SM Lagrangian without the Higgs potential. The key point of the approach under consideration is the replacement of the Higgs potential by the interaction Lagrangian

$$
L_{i n t}(\phi, H)=-\lambda\left(|H|^{2}-\frac{\xi}{M} \phi^{2}\right)^{2}
$$

of the Higgs and Goldberger-Wise fields, $\xi$ being a positive dimensionless parameter. 
The background solutions for the metric and the scalar field, which preserve the Poincaré invariance in any four-dimensional subspace $y=$ const, look like

$$
\begin{aligned}
d s^{2} & =e^{-2 A(y)} \eta_{\mu \nu} d x^{\mu} d x^{\nu}+d y^{2} \equiv \gamma_{M N}(y) d x^{M} d x^{N}, \\
\phi(x, y) & =\phi(y),
\end{aligned}
$$

$\eta_{\mu \nu}$ denoting the flat Minkowski metric, whereas the background (vacuum) solution for the Higgs field is standard

$$
H_{v a c}=\left(\begin{array}{c}
0 \\
\frac{v}{\sqrt{2}}
\end{array}\right)
$$

all the other SM fields being equal to zero.

If one substitutes this ansatz into the equations corresponding to action (19), one gets a system of differential equations for the functions $A(y)$ and $\phi(y)$, the brane scalar field potentials $V_{1,2}(\phi)$ and interaction Lagrangian (22) defining the boundary conditions for these equations on the branes. The potentials $V_{1,2}(\phi)$ are chosen so that they fix the values of the stabilizing field $\phi$ on the branes and stabilize the inter-brane distance $[12,13]$. Interaction Lagrangian (22) does not affect this stabilization mechanism, if the relation

$$
\phi(L)^{2}=\frac{M v^{2}}{2 \xi}
$$

between the values of the Higgs and stabilizing scalar field on the brane at $y=L$ is valid, which defines the vacuum value of the Higgs field. This means that in such a scenario the Higgs field vacuum expectation value, being proportional to the value of the stabilizing scalar field on the $\mathrm{TeV}$ brane, arises dynamically as a result of the gravitational bulk stabilization.

Now the linearized theory is obtained by expanding the metric, the scalar and the Higgs field in the unitary gauge around the background solution as

$$
\begin{aligned}
g_{M N}(x, y) & =\gamma_{M N}(y)+\frac{1}{\sqrt{2 M^{3}}} h_{M N}(x, y), \\
\phi(x, y) & =\phi(y)+\frac{1}{\sqrt{2 M^{3}}} f(x, y), \\
H(x) & =\left(\begin{array}{c}
0 \\
\frac{v+\sigma(x)}{\sqrt{2}}
\end{array}\right) .
\end{aligned}
$$

After substituting this representation into action (19) and keeping the terms of the second order in $h_{M N}$, $f$ and $\sigma$ one gets the Lagrangian of this action which is the standard free Lagrangian of the SM (i.e. the masses of all the SM fields are expressed in the same way as usually in terms of the vacuum value of the Higgs field and the coupling constants) together with the standard second variation Lagrangian of the stabilized RS model [14] supplemented by an interaction term of the scalar fields $f$ and $\sigma$ on the brane coming from interaction Lagrangian (22) [15].

Besides the fields $f$ and $\sigma$, there are two more scalar fields in the linearized theory, - the fields $h_{44}(x, y)$ and $\gamma^{\mu v} h_{\mu v}(x, y)$. However, the fields $f(x, y), h_{44}(x, y)$ and $\gamma^{\mu v} h_{\mu v}(x, y)$ are not independent: they are connected by the equations of motion of the linearized theory and a gauge condition $[14,16]$. For this reason we can use any one of them to describe the scalar states.

It turns out to be convenient to use the field $h_{44}(x, y)$. This field can be expanded in KK modes, the lowest mode $\phi_{1}(x)$ is called the radion field and the modes $\phi_{n}(x), \quad n>1$ belong to its KK tower. This expansion induces the corresponding expansion of the bulk scalar field $f(x, y)$. Substituting the latter expansion into the second variation Lagrangian and integrating over the extra dimension coordinate, one gets an effective four-dimensional Lagrangian. In case the Higgs and the radion masses are much 
lower than the masses of the radion excitations on can pass to a low energy approximation in the four-dimensional Lagrangian by integrating out the radion excitation fields. This gives an effective Lagrangian for the interactions of the Higgs and radion fields with the SM fields. However, due to the Higgs-radion mixing terms the fields $\sigma(x)$ and $\phi_{1}(x)$ are not mass eigenstates.

The physical mass eigenstate fields $h(x), r(x)$ are, as usually, obtained by a rotation diagonalizing the mass matrix

$$
\begin{aligned}
& h(x)=\cos \theta \sigma(x)+\sin \theta \phi_{1}(x) \\
& r(x)=-\sin \theta \sigma(x)+\cos \theta \phi_{1}(x) .
\end{aligned}
$$

The field $h(x)$ is the Higgs-dominated field and the field $r(x)$ is the radion-dominated field. Finally, one gets the effective interaction Lagrangian of the physical scalar fields $h(x)$ and $r(x)$ with the Standard Model fields in the following form [15]:

$$
\begin{aligned}
L_{h-r} & =-\frac{1}{2} \partial_{\mu} h(x) \partial^{\mu} h(x)-\frac{1}{2} m_{h}^{2} h^{2}(x)-\frac{1}{2} \partial_{\mu} r(x) \partial^{\mu} r(x)-\frac{1}{2} \mu_{r}^{2} r^{2}(x) \\
& -\frac{(c \cos \theta+\sin \theta)}{\Lambda_{r}} h(x)\left(T_{\mu}^{\mu}+\Delta T_{\mu}^{\mu}\right)+\frac{(c \sin \theta-\cos \theta)}{\Lambda_{r}} r(x)\left(T_{\mu}^{\mu}+\Delta T_{\mu}^{\mu}\right)- \\
& -\sum_{f} \frac{m_{f}}{v} \bar{\psi}_{f} \psi_{f}(\cos \theta h(x)-\sin \theta r(x))-\frac{2 M_{W}^{2}}{v}\left(W_{\mu}^{-} W^{\mu+}\right)(\cos \theta h(x)-\sin \theta r(x))+ \\
& -\frac{M_{Z}^{2}}{v}\left(Z_{\mu} Z^{\mu}\right)(\cos \theta h(x)-\sin \theta r(x))-\frac{M_{W}^{2}}{v^{2}}\left(W_{\mu}^{-} W^{\mu+}\right)(\cos \theta h(x)-\sin \theta r(x))^{2}+ \\
& -\frac{M_{Z}^{2}}{2 v^{2}}\left(Z_{\mu} Z^{\mu}\right)(\cos \theta h(x)-\sin \theta r(x))^{2} .
\end{aligned}
$$

Here $m_{h}^{2}$ and $m_{r}^{2}$ are the masses of the fields $h(x)$ and $r(x), \Lambda_{r}$ is the (inverse) coupling constant of the radion to the trace of the SM energy-momentum tensor, $\Delta T_{\mu}^{\mu}$ is the conformal anomaly of massless vector fields explicitly given by

$$
\Delta T_{\mu}^{\mu}=\frac{\beta\left(g_{s}\right)}{2 g_{s}} G_{\rho \sigma}^{a b} G_{a b}^{\rho \sigma}+\frac{\beta(e)}{2 e} F_{\rho \sigma} F^{\rho \sigma}
$$

with $\beta$ being the well-known QCD and QED $\beta$-functions.

The parameter $c$ accommodates the contributions of the integrated out heavy scalar modes and is expressed in terms of the physical masses and the mixing angle as follows:

$$
c=\frac{\left(m_{r}^{2}-m_{h}^{2}\right) \sin 2 \theta}{m_{r}^{2} \cos ^{2} \theta+m_{h}^{2} \sin ^{2} \theta}\left(\sum_{n=2}^{\infty} \alpha_{n}^{2}\right) .
$$

It also depends on the sum of the coefficients $\alpha_{n}^{2}$, where $\alpha_{n}$ is the ratio of the wave functions in the extra dimension of the modes $\phi_{n}$ and $\phi_{1}$ taken at $y=L$. These ratios are, of course, model dependent and should fall off with $n$ in order for the sum to be convergent. In certain models several first ratios may be of the order of unity [14]. Thus, one can conservatively estimate this sum to be also of the order of unity.

The effective four-dimensional interaction Lagrangian (30) expressed in terms of the physical Higgs-dominated $h(x)$ and radion-dominated $r(x)$ fields involves only five extra parameters in addition to those of the SM: the masses of the Higgs-dominated and radion-dominated fields $m_{h}$ and $m_{r}$, the 
mixing angle $\theta$, the (inverse) coupling constant of the radion to the trace of the energy-momentum tensor of the SM fields $\Lambda_{r}$ and the parameter $c$.

Let us consider several interesting subspaces of the parameter space of the effective theory. If we put the parameters $c$ and $\theta$ equal to zero, i.e. consider the case of the zero mixing, Lagrangian (30) becomes just the SM Higgs Lagrangian plus the usual Lagrangian of the radion interaction with the trace of the SM energy-momentum tensor. In paper [17] it was shown that this interaction Lagrangian leads to the same production and decay properties of a single radion as those of the Higgs boson. If we put $1 / \Lambda_{r}=0$, we get the effective Lagrangian of the real Higgs singlet extension of the Standard Model $[18,19]$. If we formally put the parameter $c$ equal to zero while keeping the mixing angle $\theta$ nonzero, we obtain an effective interaction Lagrangian which is very similar to the one of the unstabilized RS model with the Higgs-curvature term on the brane [20]. In particular, the couplings of the Higgsdominated and the radion-dominated states to the conformal anomaly of massless vector fields, which turn to be very important for their production and decay to two photons, are the same. However, one should keep in mind that the observable parameters of the effective Lagrangian in different cases depend differently on the fundamental parameters of the models.

Thus, Lagrangian (30) is a very general effective Lagrangian of the interaction of two mixed scalar states with the fermion and vector fields of the Standard Model extended by a singlet scalar. The extra parameters of this Lagrangian are natural for considering the phenomenology of the mixed scalar states and allow one to compare easily the predictions of different models. In fact, it is unimportant, how they depend on the fundamental parameters of a particular model as long as the latter belong to the phenomenologically acceptable parameter subspace.

In the general case of a non-zero mixing, when all the parameters and $\theta, 1 / \Lambda_{r}$, and $c$ are not equal to zero, the additional terms in the Lagrangian, coming from the integrated out heavy modes and containing the parameter $c$ that depends on the scalar state masses and the mixing angle, may lead to certain changes in the collider phenomenology of the Higgs-dominated and radion-dominated states as was demonstrated in [15], where also the Feynman rules for the model are explicitly given.

\section{Restrictions on the radion mass}

Since there are two light particles in the scalar sector, we have studied two a priori possible scenarios that the scalar state discovered at $125 \mathrm{GeV}$ is either a Higgs-dominated state or a radion-dominated state [15]. In this analysis we used only two signal strengths corresponding to the channels $g g->\gamma \gamma$ and $g g->Z Z^{*}$. The analysis showed that the interpretation of the $125 \mathrm{GeV}$ scalar state as a Higgsdominated state is the preferred one. Then the restrictions on the mass of the radion-dominated state and the mixing angle can be demonstrated by Fig. 1

We see that the most probable values of the radion-dominated state masses are close to the mass of the Higgs-dominated state. Nevertheless, there is an allowed region of heavier masses of the radiondominated state, where its production rate is decreased so that the corresponding state may be again allowed. Obviously, if one considers larger values of the parameter $\Lambda_{r}$, the cross section of the radiondominated state gets smaller and the allowed region for such a state is increased. This is demonstrated in Fig. 2, where the parameter $\Lambda_{r}$ is chosen to be $5 \mathrm{GeV}$ with all the other parameters being the same as for the considered $3 \mathrm{TeV}$ case.

Next we have studied the impact of the constraints from the measurements of the parameters of the observed $125 \mathrm{GeV}$ Higgs boson and from the unconfirmed $750 \mathrm{GeV}$ diphoton excess in the LHC experiments on the properties of a radion-dominated state [21]. It turned out that the allowed region of heavier masses of the radion-dominated state shrinks even more. In Fig. 3 it is shown that the allowed masses are now of the order of $1 \mathrm{TeV}$. 


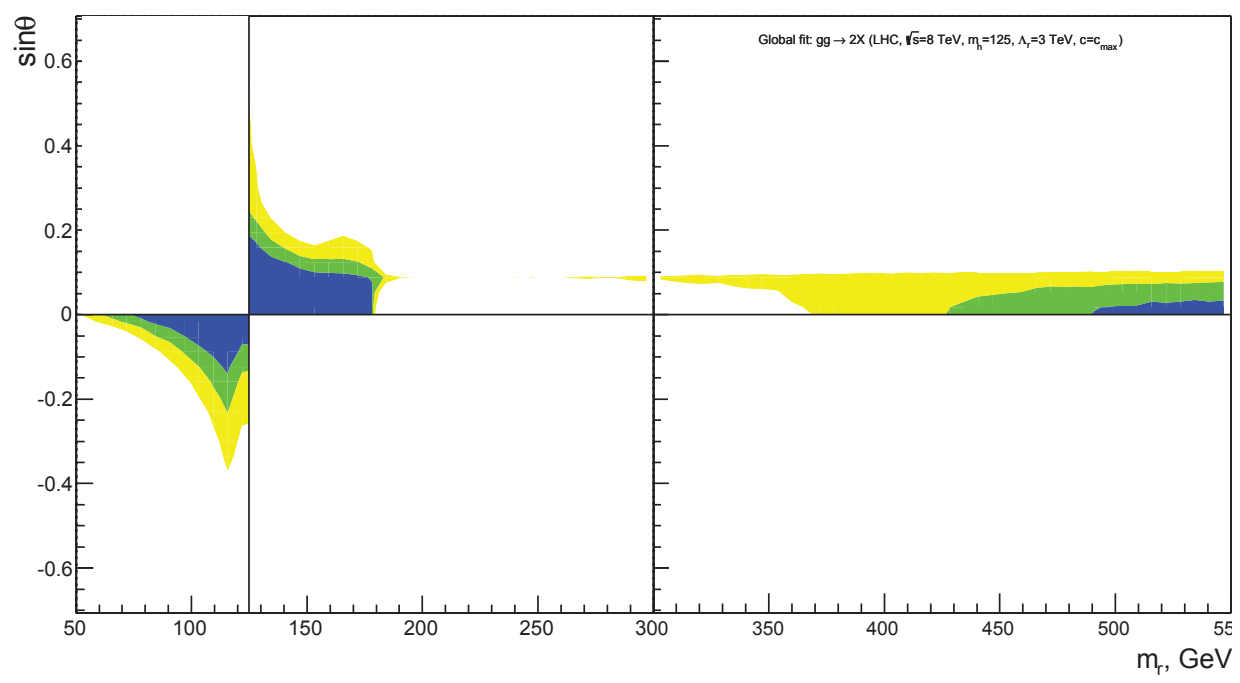

Figure 1. Exclusion contours for the combined $\chi^{2}$ fit in the $\left(m_{r}, \sin \theta\right)$ plane that includes both channels $g g \rightarrow \gamma \gamma$ and $g g \rightarrow Z Z^{*}$ for the LHC at $\sqrt{s}=8 T e V$ and $m_{h}=125, \Lambda_{r}=3 \mathrm{TeV}, c=c_{\max }$. The dark, medium and light shaded areas correspond to CL of the fit $65 \%, 90 \%$ and $99 \%$ respectively.

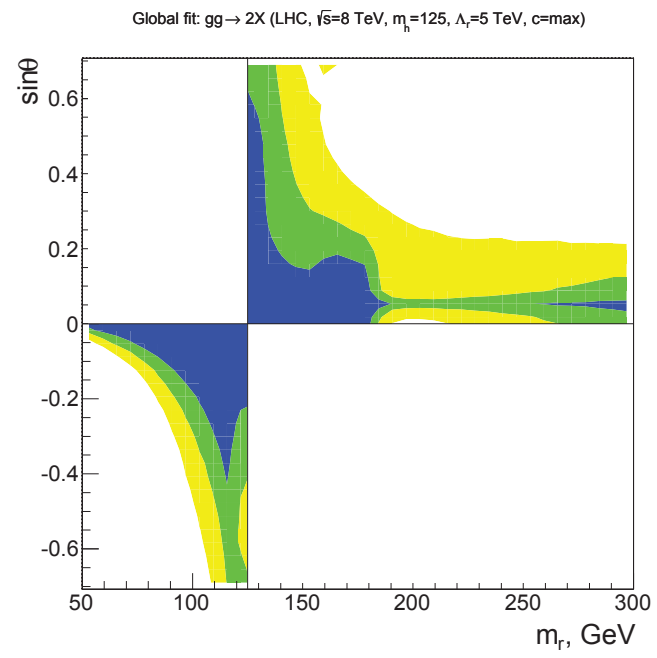

Figure 2. Exclusion contours for the combined $\chi^{2}$ fit in the $\left(m_{r}, \sin \theta\right)$ plane that includes both channels $g g \rightarrow \gamma \gamma$ and $g g \rightarrow Z Z^{*}$ for the LHC at $\sqrt{s}=8 T e V$ and $m_{h}=125, \Lambda_{r}=5 T e V, c=c_{\max }$. The dark, medium and light shaded areas correspond to CL of the fit $65 \%, 90 \%$ and $99 \%$ respectively.

However, there remain masses of the radion-dominated state below $125 \mathrm{GeV}$. The available data from the LHC also give restrictions on the model parameters in this region. We have studied in 


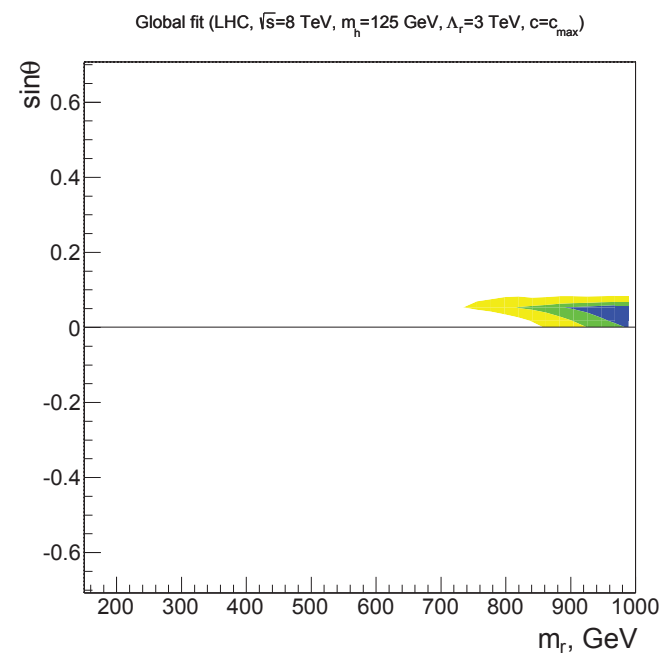

Figure 3. The region in the $\left(m_{r}, \sin \theta\right)$ still allowed by the common fit of the CMS and ATLAS exclusion limits for heavy Higgs searches and restictions from the fit of the influence of that parameters on the signal strengthes at the point $125 \mathrm{GeV}$. The dark, medium and light shaded areas correspond to $\mathrm{CL}$ of the fit $65 \%, 90 \%$ and $99 \%$ respectively. (The figure is taken from paper [15])

detail two possible values of masses below $125 \mathrm{GeV},-30 \mathrm{GeV}$ and $60 \mathrm{GeV}$ that have already been discussed in the literature. The LHC data at $8 \mathrm{TeV}$ give the restrictions on the model parameters for these radion-dominated state masses presented in Fig. 4
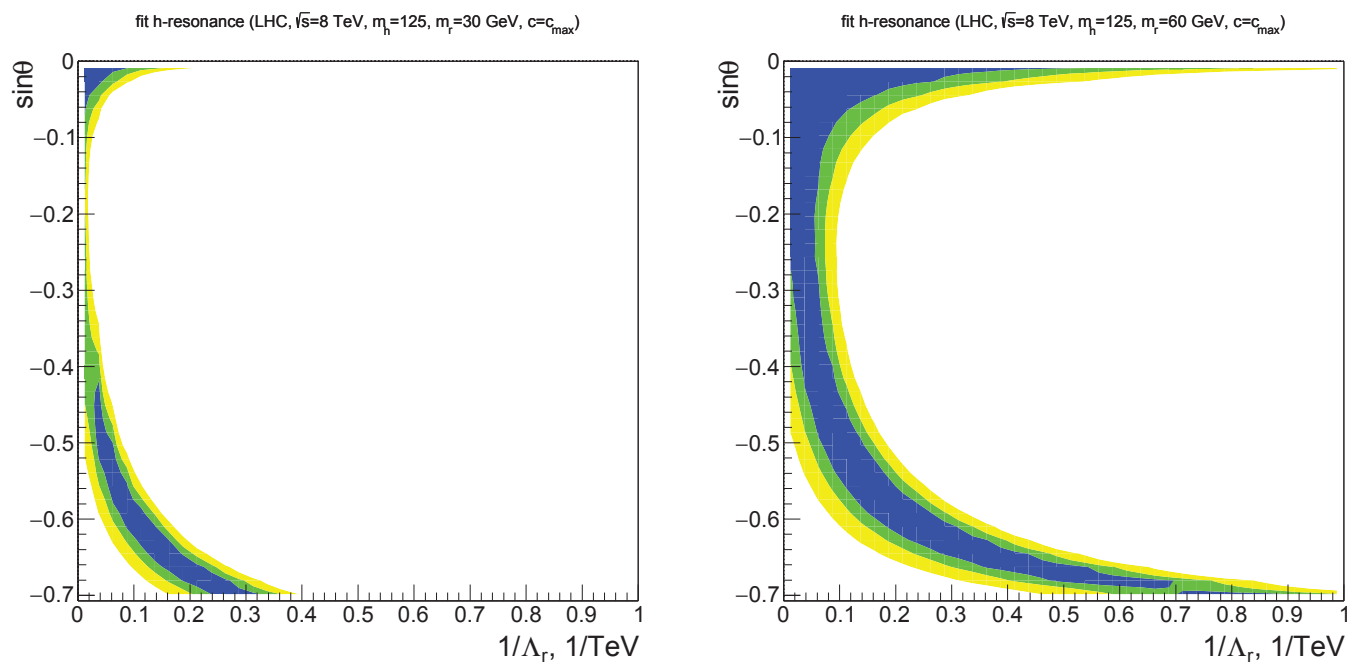

Figure 4. Exclusion contours for the partial $\chi^{2}$ fit in the $\left(\sin \theta, 1 / \Lambda_{r}\right)$ plane for the LHC at $\sqrt{s}=8 \mathrm{TeV}$ and $m_{r}=30 \mathrm{GeV}$ (left) and $m_{r}=60 \mathrm{GeV}$ (right). The dark, medium and light shaded areas correspond to CL of the fit $65 \%, 90 \%$ and $99 \%$ respectively. 
These restrictions become even stronger, if one takes into account the old LEP data. In fact, they almost exclude the existence of a light radion-dominated state for $\Lambda_{r}<10 \mathrm{TeV}$.

\section{Conclusion}

Theories with extra dimensions have a long history characterized by alternating periods of oblivion and keen interest. The latest developments in this field have put an end to the old restrictions on the size of the extra dimensions, and now their predictions can be confronted with experiment.

We believe that nowadays the most consistent model with extra dimensions is the stabilized Randall-Sundrum model. If its fundamental parameters lie in the $\mathrm{TeV}$ energy range for the observer on the negative tension brane, the effects due to the massive modes can be observed in collider experiments. In particular, the higher excitations of the scalar radion field can influence the properties of the Higgs-dominated state at $125 \mathrm{GeV}$, whereas the restrictions on the model parameters imposed by the experimental study of the properties of $125 \mathrm{GeV}$ scalar boson move the mass of the radion-dominated state to $1 \mathrm{TeV}$ or higher.

\section{References}

[1] Bernhard Riemann, Nature 8 14-17, 36, 37 (1873).

[2] G. Nordström, Physikalische Zeitschrift 15 504-506 ( 1914) [physics/0702221].

[3] T. Kaluza, Sitzungsber. Preuss. Akad. Wiss, Berlin, Math. Phys. H1, 966-972 (1921).

[4] O. Klein, Z. Phys. 37, 895-906 (1926).

[5] A. Einstein and P. Bergmann, Annals Math. 39683 (1938)

[6] Yu.A. Kubyshin, I.P. Volobuev, J. M. Mourao and G. Rudolph, Lecture Notes in Physics, 349 (1990).

[7] V.A. Rubakov, M.E. Shaposhnikov, Phys. Lett. B125 136 (1983).

[8] N. Arkani-Hamed, S. Dimopoulos, G. Dvali, B429 263 (1998) [hep-ph/9803315].

[9] L. Randall, R. Sundrum, Phys. Rev. Lett. 833370 (1999) [hep-ph/9905221].

[10] Sean M. Carroll, Monica M. Guica, hep-th/0302067.

[11] M. N. Smolyakov, Class. Quantum Grav. 25238003 (2008) [gr-qc/0811. 0299].

[12] W. D. Goldberger, M. B. Wise. Phys. Rev. Lett. 834922 (1999) [hep-ph/9907447].

[13] O. DeWolfe, D. Z. Freedman, S. S. Gubser, A. Karch, Phys. Rev. D. V. 62046008 (2000) [hep-th/9909134].

[14] E. E. Boos, Yu. S. Mikhailov, M. N. Smolyakov, I. P. Volobuev, Mod. Phys. Lett. A 21, 14311449 (2006) [hep-th/0511185].

[15] E. E. Boos, V. E. Bunichev, M. A. Perfilov, M. N. Smolyakov and I. P. Volobuev, Phys. Rev. D 92 (2015) no.9, 095010 [arXiv: 1505.05892].

[16] C. Csaki, M. L. Graesser and G. D. Kribs, Phys. Rev. D 63, 065002 (2001) [hep-th/0008151].

[17] E. Boos, S. Keizerov, E. Rahmetov and K. Svirina, Phys. Rev. D 90, 095026 (2014) [arXiv: 1409.2796].

[18] S. I. Godunov, A. N. Rozanov, M. I. Vysotsky and E. V. Zhemchugov, arXiv: 1503.01618.

[19] T. Robens and T. Stefaniak, Eur. Phys. J. C 75 (2015) 104 [arXiv: 1501 . 02234].

[20] G. F. Giudice, R. Rattazzi and J. D. Wells, Nucl. Phys. B 595, 250 (2001) [hep-ph/0002178].

[21] E. E. Boos, V. E. Bunichev and I. P. Volobuev, J. Exp. Theor. Phys. 124 (2017) no.5, 722. 